\title{
MULTI-FEATURE-MARKS BASED INFORMATION EXTRACTION OF URBAN GREEN SPACE ALONG ROAD
}

\author{
H. H. Zhao, H.Y. Guan
}

Nanjing University of Information Science and Technology, Nanjing, China - haohao_zhao@hotmail.com

KEY WORDS: High resolution remote sensing imagery, Urban green space along road, Spectrum features, Recognition marks, Multi-feature-marks segmentation, Information Extraction

\begin{abstract}
:
Green space along road of QuickBird image was studied in this paper based on multi-feature-marks in frequency domain. The magnitude spectrum of green along road was analysed, and the recognition marks of the tonal feature, contour feature and the road were built up by the distribution of frequency channels. Gabor filters in frequency domain were used to detect the features based on the recognition marks built up. The detected features were combined as the multi-feature-marks, and watershed based image segmentation were conducted to complete the extraction of green space along roads. The segmentation results were evaluated by Fmeasure with $P=0.7605, R=0.7639, \quad F=0.7622$.
\end{abstract}

\section{INTRODUCTION}

Green space along urban road plays an important part of urban vegetation, which has been the key factor influencing on urban ecological, microclimate, and living comfort of urban dwellers. The extraction of urban green space along road is important for urban construction and management. With the improving of spatial resolution of remote sensing images and the lasting needs of detailed data for urban management, remote sensing of urban green has gradually transited from image pixel based image classification to objects oriented feature detection. At the same time, features such as structure, semantics, topology and background have been paid more and more attention, and multifeatures based information extraction methods in spatial domain has been proposed by researchers (Wen et al., 2017; Li et al., 2014; Zhou et al., 2015).

While image features could also be reflected in the frequency domain. Such as the tonal features can be described by low frequency channels, and the contour features can be expressed by high frequency channels (Zhao et al., 2013). Then in this paper, the features of urban green space along roads are reflected by particular frequency channels, and are extracted though frequency filtering to be the feature labels, which are used to help image segmentation by watershed transformation.

The remainder of this paper is organized as follows: Sec. 2 presents the methodology for the feature recognition and information extraction, Sec. 3 discusses the experimental results. Sec. 4 gives the concluding remarks.

\section{METHODS}

\subsection{Feature Recognition}

Firstly the remotely sensed images were Fourier transformed, the magnitude spectrum of green along road was analysed, the distribution feature at specific directions and frequencies were in depth exploration, and the recognition marks for tonal feature in low frequency, for contour feature in high frequency and for the feature of road in medium frequency were built up.
2.1.1 Recognition of Tonal Feature: Spectrum images show that the main structure and tonal feature can be reflected by the low frequency channel. Firstly, the energy percent of different frequency channels was computed to analysis the most important low frequency channel for tonal information. If the total energy of the image is $E$, and the energy of the frequency channel $f_{c}$ is $E_{c}$, then the percentage of the energy channel $E_{p}$ is equal to $E_{c} / E$. Always, in the very low frequency channel, $E_{p}$ develops sharply. The higher the frequency $f_{c}$, the slower $E_{p}$ changes. The $60 \%$ energy always gathers in the very short low frequency channel, which could describe the most important tonal information of the images, and then we focus the low frequency channel in $E_{p}<60 \%$.

The green space along road has its' direction, which is the same with the road. Direction features can be easily recognizes by the spectrum images based on the spectrum bright line. And then the magnitude in different direction was analysed, the frequency $f_{l}$ with peak energy in the low frequency channel $f_{c}$ was chosen to be the mark for tonal recognition, which will be used for the filtering in Sec. 2.2.

2.1.2 Recognition of Contour Feature: Different with tonal feature, contour feature mainly distributes in the high frequency channel. While the high frequency channels always be long, it's difficult to find the recognition marks in the magnitude curve directly. To choose the optimal frequency mark for describing the contour feature of the image, we compute the first order derivative of the magnitude. If the magnitude in the direction $\theta$ is $S_{\theta}$, the first order derivative of $S_{\theta}$ is $S_{\theta}^{\prime}$, and $S_{\theta}^{\prime}$ is further analysed for choose the recognition mark.

In 2.1.1, we discuss the low frequency channel of $E_{p}<60 \%$, here we focus the frequency channel of the remnant $40 \%$ in the higher frequency channels. The first order derivative of a function represents the speed of the variable, that is, $S_{\theta}^{\prime}$ can reflect the speed of the energy developing with the frequency in some direction. If $S_{\theta}^{\prime}$ is higher, the energy changes more quickly, which means there is greater difference and variation in the image structure, that is fit for the feature of contour. Then 
we choose the frequency $f_{h}$ with the highest $S_{\theta}^{\prime}$ as the recognition mark for the contour.

2.1.3 Feature Recognition of Road: In remotely sensed images, roads have their directions, which can be recognized by the angular distribution curve of the spectrum images. For the recognition of green space along road, we focus on the centre line of the road. We have discussed that the low and high frequency channel for the tonal and contour features of the image. While for the texture of the road, it doesn't distribute in the very lower frequency channel, because there is vogue boundary in it. And nor in the higher frequency channel, because there is mainly edge features in it, and we can't detect the centre line by the edges.

Then we firstly compute the angular distribution curve of the spectrum images, and the direction with peak value energy is as the direction of the road. For every direction, we analyse the most significant frequency channel between the lower frequency mark $f_{l}$ and the higher frequency mark $f_{h}$. As the texture feature of road is obvious, and we concern mainly about the centre line assisting the green space, here we give an experienced reference frequency value $f_{m}$ locating at the energy percentage $E_{p}=80 \%$, which is as the recognition mark in the medium frequency channel for the recognition of road.

\subsection{Information Extraction}

Basing on the frequency domain filtering, tonal features in low frequency, contour features in high frequency, and the supplementary information about roads in medium frequency was obtained by Gabor filters. In the end, the tonal features, contour features and road information were combined as the multi-feature-marks, watershed based image segmentation were conducted to complete the extraction of green space along roads.

2.2.1 Frequency Filtering: Gabor filters in frequency domain are used for feature detection based on the feature marks (Mehrotra et al., 1992). A 2D Gabor filter is defined by:

$$
h(x, y)=g\left(x^{\prime}, y^{\prime}\right) \exp \left[2 \pi j\left(U_{x}, V_{y}\right)\right]
$$

where, $\left(U_{x}, V_{y}\right)$ are the coordinates of the central frequency; $g(x, y)$ is the Gaussian function; $\left(x^{\prime}, y^{\prime}\right)$ are $(x, y)$ rotated by $\theta$. The 2D Gabor filter is derived by:

$$
h(x, y)=R(x, y)+j I(x, y)
$$

where,

$$
\begin{aligned}
& R(x, y)=g(x, y) \cos [\varpi(x \cos \theta+y \sin \theta)] \\
& I(x, y)=g(x, y) \sin [\varpi(x \cos \theta+y \sin \theta)]
\end{aligned}
$$

where, $\varpi$ is the angular frequency.

The real part of the Gabor filter, $R(x, y)$ is an even function, which can be defined in frequency domain by:

$$
\begin{aligned}
& G_{e}\left(u, v, \sigma_{u}, \sigma_{v}, r\right)=\frac{1}{2}\left\{\exp \left[-\left(u^{\prime}+r\right)^{2} / 2 \sigma_{u}{ }^{2}\right]+\exp \left[-\left(u^{\prime}-r\right)^{2} / 2 \sigma_{u}{ }^{2}\right]\right\} \\
& \exp \left[-\left(v^{\prime}-r\right)^{2} / 2 \sigma_{v}{ }^{2}\right]
\end{aligned}
$$

Where, $\left(u^{\prime}, v^{\prime}\right)=(u \cos \theta+v \sin \theta,-u \cos \theta+v \sin \theta), r$ is the central frequency, $\sigma_{u}$ and $\sigma_{v}$ are the spatial scales. By specific central frequency filtering, we can get smooth feature of the images. Here the even Gabor filter is used for detection the tonal feature and the road. For the parameters in $G_{e}, r$ is defined as the recognition frequency marks, $\theta$ is defined by directions of the roads. To emphasize the linear character, here we define the $\sigma_{u} / \sigma_{v}=2$.

And for the tonal feature, based on the recognition mark, there is a harmonic wave band, in which the mark is at the peak location. Then we define $\sigma_{u}$ as half of the harmonic wave band.

As for the detection of the road centre line, we define $\sigma_{u}=f_{m}-f_{l}$, and also $\sigma_{u} / \sigma_{v}=2$. The texture feature of the road can be detected by the even Gabor filter, and by binary segmentation and ridge extraction, we get the centre lines of the roads.

The imaginary part of the Gabor filter, $I(x, y)$-- an odd function -- has been shown to be an efficient and robust edge detector(reference), then we use it to detect the contour feature by the recognition marks in high frequency. In frequency domain, $I(x, y)$ is defined as:

$$
\begin{aligned}
& G_{o}\left(u, v, \sigma_{u}, \sigma_{v}, r\right)=\frac{1}{2} j \times \exp \left\{-\left[\left(u^{\prime}-r\right)^{2} / 2 \sigma_{u}^{2}+\left(v^{\prime}-r\right)^{2} / 2 \sigma_{v}^{2}\right]\right\} \\
& -\frac{1}{2} j \times \exp \left\{-\left[\left(u^{\prime}+r\right)^{2} / 2 \sigma_{u}^{2}+\left(v^{\prime}+r\right)^{2} / 2 \sigma_{v}^{2}\right]\right\}
\end{aligned}
$$

Here we define $r$ as the frequency of the contour recognition mark in 2.1.2. For the spatial scales $\sigma_{u}$ and $\sigma_{v}$, we define them by the range and angular distribution curve. In the range distribution curve, we compute the variance at the centre of $f_{h}$, the frequency radius with maximum variance is defined as $\sigma_{u}$. In the angular distribution curve, we compute the variance centring at the direction with peak value energy. If the angular radius $\theta_{v}$ has the maximum variance value, $\sigma_{v}=f_{h} \tan \theta_{v}$.

2.2.2 Multi-Feature Marked Watershed Transform: In Sec. 2.2.1, Gabor filters in frequency domain are adopted to acquire features about green space along road, then the tonal feature, contour feature and road centre line are detected. Here we extract urban green along road by the method of multifeature marked watershed transforming.

Firstly, for the tonal feature image after even Gabor filtering, it is binary segmented. The green space in the image will be highlighted, while other ground objects are dark. To remove green space which is not adjacent to the road, the road centre line is also as a label for buffer analysis in the next step. The buffer distance is based on the survey of the study area. Then we can acquire the tonal label of green space along road.

Base on the tonal label, the contour feature image is watershed transformed to finish the segmentation of the green space along road. For the height $h$ in watershed, the smaller of the value, the more fragment in the segmentation. And then a suitable $h$ for the watershed is important, in our test data, we define $h$ an experienced value as 43 . 


\section{RESULTS AND DISCUSSION}

\subsection{Testing Data}

The test dataset was selected from QuickBird images located in Nanjing, China. The spatial resolution is $0.61 \mathrm{~m}$ after fusing

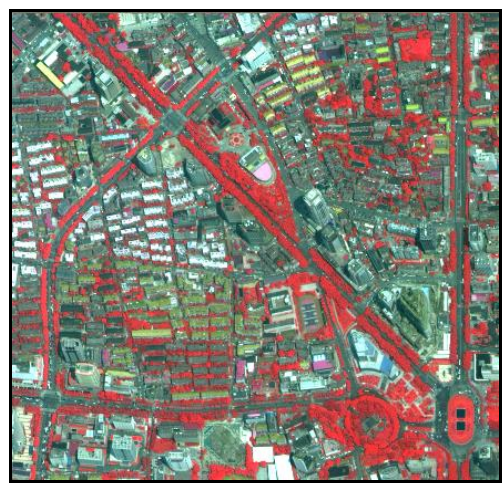

(a) multispectral bands and panchromatic bands. Near-infrared band image was chosen because its' spectral character was the most suitable for green space analysis. The image size of $1686 \times 1766$ pixels, and covered about $1.11 \mathrm{~km}^{2}$. There are 5 roads in the research area with different directions. The false color image and NDVI image are shown in Fig.1.

Figure 1. Test dataset ((a) is the false color image and (b) is the NDVI image)

\subsection{Results of Feature Detection}

The Gabor filters in frequency domain were used to detect the features of the green space along road. Tonal feature, contour

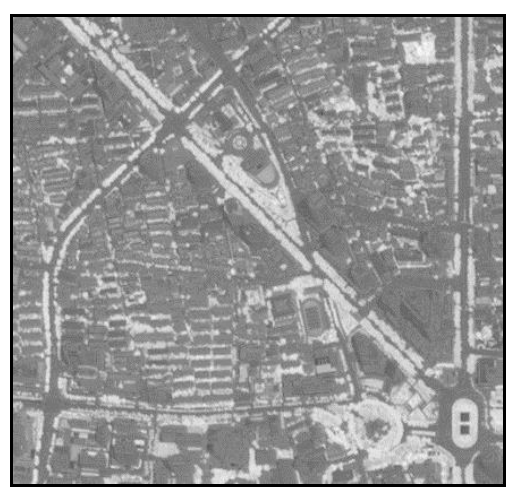

(b)
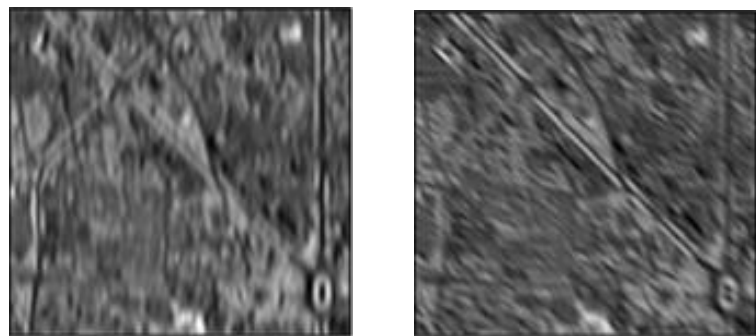

feature and road centre lines are recognized. Features in 4 main directions are shown in Fig. 2.
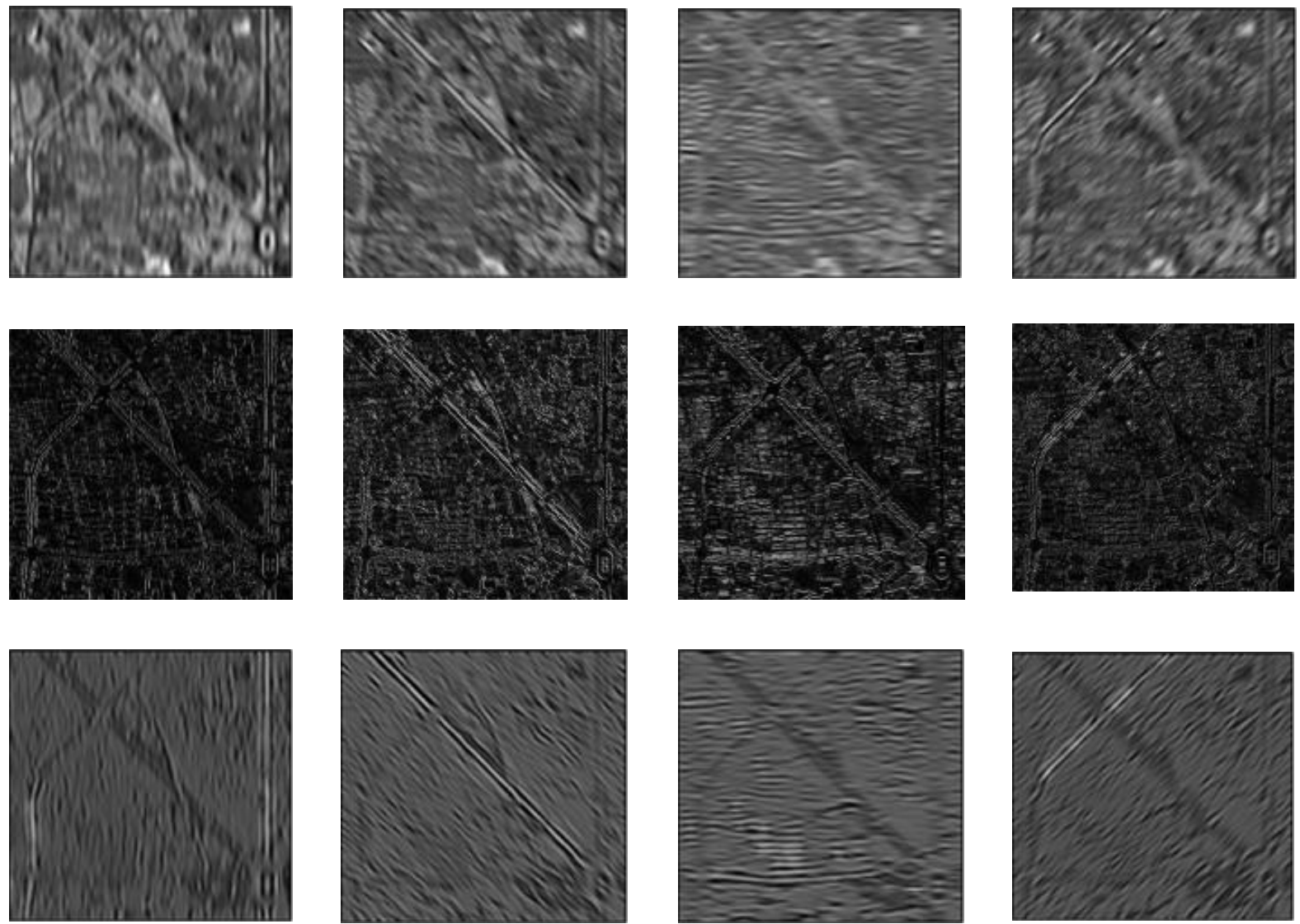

Figure 2. Features detected by Gabor filters(the top are the low frequency features, the middle are the high frequency features, and the upper are the medium frequency features) 
As we seen in Fig.2., the low frequency by Gabor filters could detect the tonal of the green space, but with vague edges. The high frequency filtering results could detect the contour features mainly of the green space along roads, and carrying some noise together. The medium frequency filtering results could detect the road features.
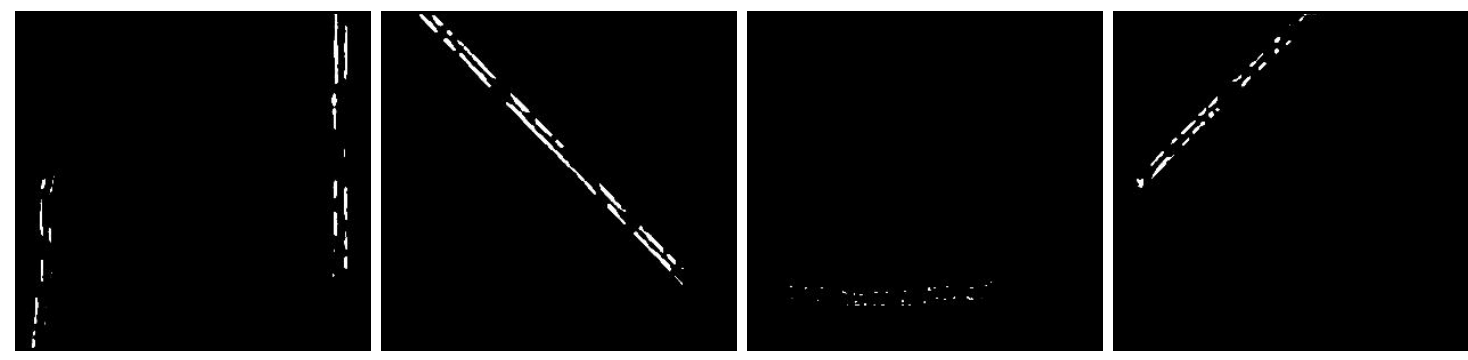

Figure 3. Labels of tonal features

Next, binarization was done by the threshed value on the tonal and road features. And the buffer was analysed to get the label of green space along road, Fig.3. shows the labels.

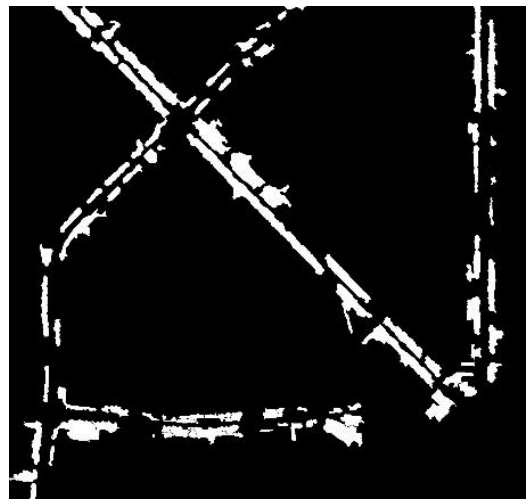

The contour features were watershed segmented based on the labels in Fig. 3., the results are shown in Fig. 4. The green space along road are detected completely, and the boundaries are smooth and clear.

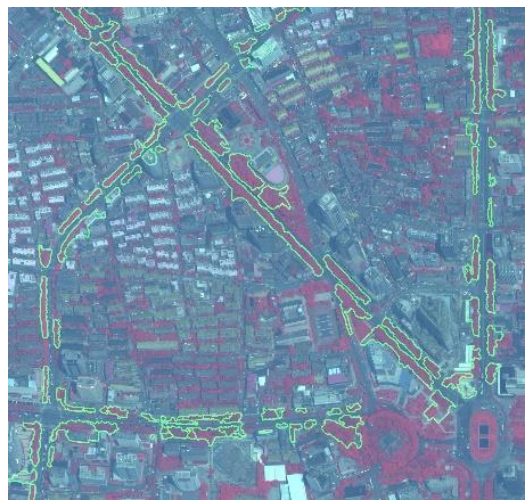

Figure 4 . The watershed segmentation results by the labels

\subsection{Precision Evaluation}

To evaluate the detection results of the green space along road, we take the measures of completeness $(P)$, correctness $(R)$, and $F$-measure (Martin, 2004). $P$ describes how complete the extracted edges are; $R$ indicates what percentage of the extracted edges are valid. F-measure is an overall score, defined as follows:

$$
F \text {-measure }=P R /(\alpha P+(1-\alpha) R)
$$

In our results, the measures are: $P=0.7605, R=0.7639, F=$ 0.7622 . We can get a relatively balanced precision by the multifeature marked segmentation.

\section{CONCLUSION}

Based on the image basic spatial features of urban green space along roads, the spectrum features were recognized in this paper to establish the tonal features recognition marks in low frequency and contour features recognition methods in high frequency, which provided a physical interpretation method based on frequency compositional variation for ground objects of high-resolution remote sensing signals. Based on the spectrum feature recognition theories, tonal features in low frequency, contour features in high frequency, and roads aided information in medium frequency, were detected by frequency domain filters, fusion features of them were used for extracting green space information along roads by multi-feature marks in frequency domain, in which way, the target of service driven typical urban green information extraction was achieved.

\section{ACKNOWLEDGEMENTS}

This work was supported in part by the National Natural Science Foundation of China under Grant 41501501, in part by the Natural Science Foundation of Jiangsu Province under Grant BK20151524.

\section{REFERENCES}

Wen D., Huang X., Liu H., et al., 2017. Semantic Classification of Urban Trees Using Very High Resolution Satellite Imagery. IEEE Journal of Selected Topics in Applied Earth Observations and Remote Sensing, 10(4), pp. 1413-1423.

Li X. X., Myint S.W., Zhang Y. J., et al., 2014. Object-based land-cover classification for metropolitan Phoenix, Arizona, using aerial photography. International Journal of Applied Earth Observation and Geoinformation, 33, pp. 321-330.

Zhou J. H., Qin J., Gao K., et al., 2015. Feature-location analyses for identification of urban tree species from very high resolution remote sensing data. Ecological Informatics, 29, pp. $16-24$.

Zhao H. H., Xiao P. F., Feng X. Z., 2013. Edge Detection of Street Trees in High-Resolution Remote Sensing Images Using Spectrum Features. Proc. of SPIE, 8918. 
The International Archives of the Photogrammetry, Remote Sensing and Spatial Information Sciences, Volume XLII-3, 2018 ISPRS TC III Mid-term Symposium "Developments, Technologies and Applications in Remote Sensing", 7-10 May, Beijing, China

Mehrotra R., Namuduri K. R., Ranganathan N., 1992. Gabor Filter-Based Edge Detection. Pattern Recognition, 25(12), pp. 1479-1494.

Martin D. R., Fowlkes C., Malik J., 2004. Learning to Detect Natural Image Boundaries Using Local Brightness, Color, and Texture Cues. IEEE Transactions on Pattern Analysis and Machine Intelligence, 26(5), pp. 530-548. 